\title{
Transfer matrix of linear solenoid fringe superimposed magnet
}

\author{
G. $\mathrm{Xu}$
}

Institute of High Energy Physics, Yuquan Road 19(B), Beijing 100039, China

(Received 25 February 2004; published 19 April 2004)

\begin{abstract}
The general transfer matrix of the superimposed solenoid magnet linear fringe field is given in this paper. It is an exact analytic matrix. The symplectic condition for the arbitrary shape solenoid will be discussed also.
\end{abstract}

DOI: 10.1103/PhysRevSTAB.7.044001

\section{INTRODUCTION}

In modern accelerators, solenoid and quadrupole magnets are often superposed, especially in the interaction region. The transfer matrix of superimposed solenoid and quadrupole magnets is given in [1]. More superimposed cases are given in [2]. However, the matrices in both [1] and [2] are for the delta fringe cases. The transfer matrix of the linear solenoid fringe without other components is given in [3]. In this paper we will discuss a more general case in which the field is superimposed by a linear solenoid fringe and a quadrupole. The general solution can cover the solution in [1,3] and some cases of [2]. For the case of nonlinear solenoid fringe, one can refer to $[4,5]$.

\section{MAGNETIC FIELD}

Assuming the magnetic field for the linear fringe solenoid,

$$
\begin{aligned}
& B_{x}=b_{x}+G y+R x+f(x, y, z), \\
& B_{y}=b_{y}+G x-R y+g(x, y, z), \\
& B_{z}=a+2 b z,
\end{aligned}
$$

where $b_{x}$ and $b_{y}$ are the dipole components in the horizontal and vertical planes, respectively, $G$ and $R$ are the normal and skew quadrupoles, respectively, $a$ is the longitudinal field at the start point, and $b$ is the slope of the longitudinal fringe. For the general field form of the solenoid, one can refer to [6].

Substituting (1) into the Maxwell equations, one can obtain the following equations:

$$
\begin{aligned}
\partial_{z} f(x, y, z) & =0, \\
\partial_{z} g(x, y, z) & =0, \\
-\partial_{y} f(x, y, z)+\partial_{x} g(x, y, z) & =0, \\
\partial_{x} f(x, y, z)+\partial_{y} g(x, y, z)+2 b & =0 .
\end{aligned}
$$

Assuming $f(x, y, z)$ and $g(x, y, z)$ are linearly related to $x$
PACS numbers: 41.85.Lc, 41.85.Ja

and $y$, then one can get

$$
f(x, y, z)=-b x, \quad g(x, y, z)=-b y .
$$

\section{EQUATION OF MOTION}

From $\vec{F}=q \vec{V} \times \vec{B}$, the Lorentz force is

$$
\begin{aligned}
& F_{x}=q\left(\left(-b_{y}-G x+b y+R y\right) C+(a+2 b z) \frac{d y}{d t}\right), \\
& F_{y}=q\left(\left(b_{x}+G y-b x+R x\right) C-(a+2 b z) \frac{d x}{d t}\right),
\end{aligned}
$$

where $\vec{V}=[(d x / d t),(d y / d t), C], C$ is the speed of light, and $q$ is the particle charge. The longitudinal force is not listed here supposing that its effect on the longitudinal motion can be neglected.

From $\vec{F}=m \vec{a}$, the equations of the particle motion are

$$
\gamma m_{0} \frac{d^{2} x}{d t^{2}}=F_{x}, \quad \gamma m_{0} \frac{d^{2} y}{d t^{2}}=F_{y},
$$

where $\gamma$ is the relativistic factor, and $m_{0}$ is the rest mass of the particle.

Transforming the variable time $t$ to position $z$, one can obtain

$$
\begin{aligned}
& \frac{d^{2} x}{d z^{2}}=-b_{y}-G x+(R+b) y+(a+2 b z) \frac{d y}{d z}, \\
& \frac{d^{2} y}{d z^{2}}=b_{x}+G y+(R-b) x-(a+2 b z) \frac{d x}{d z},
\end{aligned}
$$

where the factor $\left(q / \gamma m_{0} C\right)$ has been involved to $b_{x}, b_{y}, G$, $R, a$, and $b$.

\section{REDUCING THE EQUATION}

Equation (6) is an inhomogeneous differential equation. This means that the magnet is not coaxial with the solenoid field. Fortunately, all of the inhomogeneous terms and the coefficients of $x$ and $y$ are constant. Let $x=$ $X+C_{x}$ and $y=Y+C_{y}$, then $X$ and $Y$ satisfy the following equations: 


$$
\begin{aligned}
& \frac{d^{2} X}{d z^{2}}=-G X+(R+b) Y+(a+2 b z) \frac{d Y}{d z}, \\
& \frac{d^{2} y}{d z^{2}}=G Y+(R-b) X-(a+2 b z) \frac{d X}{d z}
\end{aligned}
$$

with

$$
C_{x}=\frac{b_{x}(b+R)+b_{y} G}{b^{2}-G^{2}-R^{2}}, \quad C_{y}=\frac{b_{y}(b-R)+b_{x} G}{b^{2}-G^{2}-R^{2}} .
$$

After a displacement transformation, the new Eq. (7) becomes a homogeneous differential equation, i.e., a coaxial case. Therefore the transfer matrix is based on an image axis which is different from the real axis of the solenoid. Actually, the displacement transformation is a simple way to handle the dipole terms $b_{x}$ and $b_{y}$ in Eq. (6). The ideal way should be to handle them in a Frenet-Serret coordinate system, a curved coordinate system. But in the Frenet-Serret coordinate the analytical solution for the linear fringe solenoid has not been found. The analytical solution given in [2] is only for the delta fringe case.

Further, making a rotation for $(X, Y)$, one can have

$$
X=x \cos (\alpha)+y \sin (\alpha), \quad Y=-x \sin (\alpha)+y \cos (\alpha),
$$

where $\alpha=\frac{1}{2} \arccos \left(R / \sqrt{G^{2}+R^{2}}\right)$. Then Eqs. (7) reduce to

$$
\begin{aligned}
& \frac{d^{2} x}{d z^{2}}=(b+R) y+(a+2 b z) \frac{d y}{d z}, \\
& \frac{d^{2} y}{d z^{2}}=(-b+R) x-(a+2 b z) \frac{d x}{d z},
\end{aligned}
$$

where the notation $R$ means $\sqrt{R^{2}+G^{2}}$. Equation (10) can be explained as the case of the solenoid fringe superimposed skew quadrupole magnet.

Making a displacement transformation of $z$, i.e., $z \rightarrow$ $z-a / 2 b$, Eq. (10) holds still. Actually this is equivalent to $a=0$ :

$$
\begin{aligned}
& \frac{d^{2} x}{d z^{2}}=(b+R) y+2 b z \frac{d y}{d z} \\
& \frac{d^{2} y}{d z^{2}}=(-b+R) x-2 b z \frac{d x}{d z}
\end{aligned}
$$

\section{SOLVING THE EQUATION}

Eliminating $y$ and $d y / d z$ in Eq. (11), one can get

$$
(b-R)(5 b+R) x+16 b^{2} z \frac{d x}{d z}+4 b^{2} z^{2} \frac{d^{2} x}{d z^{2}}+\frac{d^{4} x}{d z^{4}}=0 .
$$

Making a transformation for $z \rightarrow(\sqrt{2} / \sqrt{b}) s^{1 / 4}$,

$$
\begin{aligned}
\frac{(b-R)(5 b+R)}{64 b^{2}} x+\frac{3+56 s}{32} \frac{d x}{d s}+\frac{51 s+16 s^{2}}{16} \frac{d^{2} x}{d s^{2}}+ & \frac{9 s^{2}}{2} \frac{d^{3} x}{d s^{3}}+s^{3} \frac{d^{4} x}{d s^{4}}=0 .
\end{aligned}
$$

Further making a transformation for $s \rightarrow-s$,

$$
\begin{aligned}
& \frac{(R-b)(5 b+R)}{64 b^{2}} x+\frac{3-56 s}{32} \frac{d x}{d s}+\frac{51 s-16 s^{2}}{16} \frac{d^{2} x}{d s^{2}}+ \\
& \frac{9 s^{2}}{2} \frac{d^{3} x}{d s^{3}}+s^{3} \frac{d^{4} x}{d s^{4}}=0 .
\end{aligned}
$$

Actually, ${ }_{2} F_{3}\left(a_{1}, a_{2} ; \rho_{1}, \rho_{2}, \rho_{3} ; s\right)$, the generalized hypergeometric function satisfies the following function (see Ref. [7]):

$$
\begin{gathered}
-a_{1} a_{2} x+\left[\rho_{1} \rho_{2} \rho_{3}-\left(1+a_{1}+a_{2}\right) s\right] \frac{d x}{d s}+ \\
s^{2}\left(3+\rho_{1}+\rho_{2}+\rho_{3}\right) \frac{d^{3} x}{d s^{3}}+ \\
s\left(1+\rho_{1}+\rho_{2}+\rho_{3}+\rho_{1} \rho_{2}+\rho_{1} \rho_{3}+\rho_{2} \rho_{3}-s\right) \frac{d^{2} x}{d s^{2}}+ \\
s^{3} \frac{d^{4} x}{d s^{4}}=0 .
\end{gathered}
$$

Comparing Eq. (14) with (15), one can solve out

$$
\begin{aligned}
& a_{1}=\frac{b-R}{8 b}, \quad a_{2}=\frac{5 b+R}{8 b}, \quad \rho_{1}=\frac{1}{4}, \\
& \rho_{2}=\frac{1}{2}, \quad \rho_{3}=\frac{3}{4} .
\end{aligned}
$$

In fact, $s^{1-\rho 1}{ }_{2} F_{3}\left(1+a_{1}-\rho_{1}, 1+a_{2}-\rho_{1} ; 2-\rho_{1}, 1+\right.$ $\left.\rho_{2}-\rho_{1}, 1+\rho_{3}-\rho_{1} ; s\right), \quad s^{1-\rho 2} \quad{ }_{2} F_{3}\left(1+a_{1}-\rho_{2}, 1+\right.$ $\left.a_{2}-\rho_{2} ; 1+\rho_{1}-\rho_{2}, 2-\rho_{2}, 1+\rho_{3}-\rho_{2} ; s\right)$, and $s^{1-\rho 3}$ ${ }_{2} F_{3}\left(1+a_{1}-\rho_{3}, 1+a_{2}-\rho_{3} ; 1+\rho_{1}-\rho_{3}, 1+\rho_{2}-\rho_{3}, 2-\right.$ $\left.\rho_{3} ; s\right)$ are also the solutions of Eq. (15).

Substituting $s$ back to $z$, then the general solution of Eq. (12) is

$$
\begin{aligned}
x= & { }_{2} F_{3}\left(\frac{b-R}{8 b}, \frac{5 b+R}{8 b} ; \frac{1}{4}, \frac{1}{2}, \frac{3}{4} ;-\frac{b^{2} z^{4}}{4}\right) C_{1} \\
& +z_{2} F_{3}\left(\frac{3 b-R}{8 b}, \frac{7 b+R}{8 b} ; \frac{1}{2}, \frac{3}{4}, \frac{5}{4} ;-\frac{b^{2} z^{4}}{4}\right) C_{2} \\
& +z^{2}{ }_{2} F_{3}\left(\frac{5 b-R}{8 b}, \frac{9 b+R}{8 b} ; \frac{3}{4}, \frac{5}{4}, \frac{3}{2} ;-\frac{b^{2} z^{4}}{4}\right) C_{3} \\
& +z^{3}{ }_{2} F_{3}\left(\frac{7 b-R}{8 b}, \frac{11 b+R}{8 b} ; \frac{5}{4}, \frac{3}{2}, \frac{7}{4} ;-\frac{b^{2} z^{4}}{4}\right) C_{4} .
\end{aligned}
$$

Let the initial conditions for $x, x^{\prime}, y$, and $y^{\prime}$ be $x(0), x^{\prime}(0)$, $y(0)$, and $y^{\prime}(0)$, then the initial values of $x^{\prime \prime}$ and $x^{\prime \prime \prime}$ are $x^{\prime \prime}(0)=(b+R) y(0), x^{\prime \prime \prime}(0)=(3 b+R) y^{\prime}(0)$. So one can get 
$C_{1}=x(0), \quad C_{2}=x^{\prime}(0), \quad C_{3}=(b+R) y(0) / 2$,

$C_{4}=(3 b+R) y^{\prime}(0) / 6$.

If $b \rightarrow-b$ and $x \Leftrightarrow y$, Eq. (11) will keep the same form. So the solution for $y$ is

$$
\begin{aligned}
y= & { }_{2} F_{3}\left(\frac{b+R}{8 b}, \frac{5 b-R}{8 b} ; \frac{1}{4}, \frac{1}{2}, \frac{3}{4} ;-\frac{b^{2} z^{4}}{4}\right) C_{5} \\
& +z_{2} F_{3}\left(\frac{3 b+R}{8 b}, \frac{7 b-R}{8 b} ; \frac{1}{2}, \frac{3}{4}, \frac{5}{4} ;-\frac{b^{2} z^{4}}{4}\right) C_{6} \\
& +z_{2}^{2}{ }_{2} F_{3}\left(\frac{5 b+R}{8 b}, \frac{9 b-R}{8 b} ; \frac{3}{4}, \frac{5}{4}, \frac{3}{2} ;-\frac{b^{2} z^{4}}{4}\right) C_{7} \\
& +z_{2}^{3} F_{3}\left(\frac{7 b+R}{8 b}, \frac{11 b-R}{8 b} ; \frac{5}{4}, \frac{3}{2}, \frac{7}{4} ;-\frac{b^{2} z^{4}}{r}\right) C_{8},
\end{aligned}
$$

where

$$
\begin{aligned}
& C_{5}=y(0), \quad C_{6}=y^{\prime}(0), \quad C_{7}=(R-b) x(0) / 2, \\
& C_{8}=(R-3 b) x^{\prime}(0) / 6 .
\end{aligned}
$$

For the special case $R=0$,

$$
{ }_{2} F_{3}\left(\frac{1}{8}, \frac{5}{8} ; \frac{1}{4}, \frac{1}{2}, \frac{3}{4},-z^{2}\right)=\gamma\left(\frac{3}{4}\right)\left(\frac{z}{2}\right)^{1 / 4} J_{-1 / 4}(z) \cos (z)
$$

where $\gamma$ is the gamma function, and $J$ is the modified Bessel function. The solution will be reduced to the case in [2].

\section{TRANSFER MATRIX}

From Eqs. (17)-(20), one can obtain the transfer matrix $M(z, 0)$ of the particle motion from 0 to $z$ for Eq. (11):

$$
M(z, 0)=\left(\begin{array}{llll}
M_{11} & M_{12} & M_{13} & M_{14} \\
M_{21} & M_{22} & M_{23} & M_{24} \\
M_{31} & M_{32} & M_{33} & M_{34} \\
M_{41} & M_{42} & M_{43} & M_{44}
\end{array}\right),
$$

where

$$
\begin{aligned}
& M_{11}={ }_{2} F_{3}\left(\alpha_{0}^{-}, \alpha_{2}^{+} ; \frac{1}{4}, \frac{1}{2}, \frac{3}{4} ;-\frac{b^{2} z^{4}}{4}\right), \\
& M_{12}=z_{2} F_{3}\left(\alpha_{1}^{-}, \alpha_{3}^{+} ; \frac{1}{2}, \frac{3}{4}, \frac{5}{4} ;-\frac{b^{2} z^{4}}{4}\right),
\end{aligned}
$$

$$
M_{13}=4 b z_{2}^{2} F_{3}\left(\alpha_{2}^{-}, \alpha_{4}^{+} ; \frac{3}{4}, \frac{5}{4}, \frac{3}{2} ;-\frac{b^{2} z^{4}}{4}\right) \alpha_{0}^{+},
$$

$$
M_{14}=\frac{4}{3} b z_{2}^{3} F_{3}\left(\alpha_{3}^{-}, \alpha_{5}^{+} ; \frac{5}{4}, \frac{3}{2}, \frac{7}{4} ;-\frac{b^{2} z^{4}}{4}\right) \alpha_{1}^{+} \text {, }
$$

$$
M_{21}=\frac{-32}{3} b^{2} z_{2}^{3} F_{3}\left(\alpha_{4}^{-}, \alpha_{6}^{+} ; \frac{5}{4}, \frac{3}{2}, \frac{7}{4} ;-\frac{b^{2} z^{4}}{4}\right) \alpha_{0}^{-} \alpha_{2}^{+},
$$

$$
\begin{aligned}
& M_{22}={ }_{2} F_{3}\left(\alpha_{1}^{-}, \alpha_{3}^{+} ; \frac{1}{2}, \frac{3}{4}, \frac{5}{4} ;-\frac{b^{2} z^{4}}{4}\right)-\frac{32}{15} b^{2} z_{2}^{4} F_{3}\left(\alpha_{5}^{-}, \alpha_{7}^{+} ; \frac{3}{2}, \frac{7}{4}, \frac{13}{4} ;-\frac{b^{2} z^{4}}{4}\right) \alpha_{1}^{-} \alpha_{3}^{+}, \\
& M_{23}=8 b z_{2} F_{3}\left(\alpha_{2}^{-}, \alpha_{4}^{+} ; \frac{3}{4}, \frac{5}{4}, \frac{3}{2} ;-\frac{b^{2} z^{4}}{4}\right) \alpha_{0}^{+}-\frac{128}{45} b^{3} z_{2}^{5} F_{3}\left(\alpha_{6}^{-}, \alpha_{8}^{+} ; \frac{7}{4}, \frac{9}{4}, \frac{5}{2} ;-\frac{b^{2} z^{4}}{4}\right) \alpha_{0}^{+} \alpha_{2}^{-} \alpha_{4}^{+} \text {, } \\
& M_{24}=4 b z_{2}^{2} F_{3}\left(\alpha_{3}^{-}, \alpha_{5}^{+} ; \frac{5}{4}, \frac{3}{2}, \frac{7}{4} ;-\frac{b^{2} z^{4}}{4}\right) \alpha_{1}^{+}-256 b^{3} z_{2}^{6} F_{3}\left(\alpha_{7}^{-}, \alpha_{9}^{+} ; \frac{9}{4}, \frac{5}{2} 2 \frac{11}{4} ;-\frac{b^{2} z^{4}}{4}\right) \alpha_{1}^{+} \alpha_{3}^{-} \alpha_{5}^{+} \text {, } \\
& M_{31}=-4 b z_{2}^{2} F_{3}\left(\alpha_{2}^{+}, \alpha_{4}^{-} ; \frac{3}{4}, \frac{5}{4}, \frac{3}{2} ;-\frac{b^{2} z^{4}}{4}\right) \\
& M_{32}=\frac{-4}{3} b z_{2}^{3} F_{3}\left(\alpha_{3}^{+}, \alpha_{5}^{-} ; \frac{5}{4}, \frac{3}{2}, \frac{7}{4} ;-\frac{b^{2} z^{4}}{4}\right) \alpha_{1}^{-} \\
& M_{33}={ }_{2} F_{3}\left(\alpha_{0}^{+}, \alpha_{2}^{-} ; \frac{1}{4}, \frac{1}{2}, \frac{3}{4} ;-\frac{b^{2} z^{4}}{4}\right) \text {, } \\
& M_{34}=z_{2} F_{3}\left(\alpha_{1}^{+}, \alpha_{3}^{-} ; \frac{1}{2}, \frac{3}{4}, \frac{5}{4} ;-\frac{b^{2} z^{4}}{4}\right) \\
& M_{41}=-8 b z_{2} F_{3}\left(\alpha_{2}^{+}, \alpha_{4}^{-} ; \frac{3}{4}, \frac{5}{4}, \frac{3}{2} ;-\frac{b^{2} z^{4}}{4}\right) \alpha_{0}^{-}+\frac{128}{45} b^{3} z_{2}^{5}{ }_{2} F_{3}\left(\alpha_{6}^{+}, \alpha_{8}^{-} ; \frac{7}{4}, \frac{9}{4}, \frac{5}{2} ;-\frac{b^{2} z^{4}}{4}\right) \alpha_{0}^{-} \alpha_{2}^{+} \alpha_{4}^{-} \text {, } \\
& M_{42}=-4 b z_{2}^{2} F_{3}\left(\alpha_{3}^{+}, \alpha_{5}^{-} ; \frac{5}{4}, \frac{3}{2}, \frac{7}{4} ;-\frac{b^{2} z^{4}}{4}\right) \alpha_{1}^{-}+256 b^{3} z_{2}^{6}{ }_{2} F_{3}\left(\alpha_{7}^{+}, \alpha_{9}^{-} ; \frac{9}{4}, \frac{5}{2}, \frac{11}{4} ;-\frac{b^{2} z^{4}}{4}\right) \alpha_{1}^{-} \alpha_{3}^{+} \alpha_{5}^{-} \text {, }
\end{aligned}
$$




$$
\begin{gathered}
M_{43}=\frac{-32}{3} b^{2} z^{3}{ }_{2} F_{3}\left(\alpha_{4}^{+}, \alpha_{6}^{-} ; \frac{5}{4}, \frac{3}{2}, \frac{7}{4} ;-\frac{b^{2} z^{4}}{4}\right) \alpha_{0}^{+} \alpha_{2}^{+}, \\
M_{44}={ }_{2} F_{3}\left(\alpha_{1}^{+}, \alpha_{3}^{-} ; \frac{1}{2}, \frac{3}{4}, \frac{5}{4} ;-\frac{b^{2} z^{4}}{4}\right)-\frac{128}{15} b^{2} z^{4}{ }_{2} F_{3}\left(\alpha_{5}^{+}, \alpha_{7}^{-} ; \frac{3}{2}, \frac{7}{4}, \frac{13}{4} ;-\frac{b^{2} z^{4}}{4}\right) \alpha_{1}^{+} \alpha_{3}^{-},
\end{gathered}
$$

and the notations $\alpha_{i}^{ \pm}=[(2 i+1) b \pm R / 8 b]$.

The transfer matrix from 0 to $z$ for Eq. (10) can be expressed as

$$
\begin{aligned}
M_{\mathrm{eq} 10}(z, 0) & =M_{\mathrm{eq} 11}\left(z+\frac{a}{2 b}, \frac{a}{2 b}\right) \\
& =M_{\mathrm{eq} 11}\left(z+\frac{a}{2 b}, 0\right) M_{\mathrm{eq} 11}^{-1}\left(\frac{a}{2 b}, 0\right) .
\end{aligned}
$$

\section{SYMPLECTIC CONDITION}

$M_{\text {eq11 }}(z, 0)$ is the transfer matrix related to $b$ and $R$; we write it down in the explicit form $M_{\text {eq } 11}(b, R ; z, 0)$. Let the longitudinal field be $B_{z}$ at position $z$, then $z=B_{z} / b$, and the matrix is $M_{\text {eq } 11}\left(b, R ; B_{z} / b, 0\right)$.

For the delta fringe case, $b \rightarrow \infty,-b^{2} z^{4} / 4=$ $-B_{z}^{4} / 4 b^{2} \rightarrow 0$. Since ${ }_{2} F_{3}\left(a_{1}, a_{2} ; \rho_{1}, \rho_{2}, \rho_{3} ; 0\right)$ is equal to 1 for any $a_{1}, a_{2}, \rho_{1}, \rho_{2}$, and $\rho_{3}$, one can get the matrix for delta fringe as

$$
\begin{aligned}
M_{\delta}\left(B_{z}, 0\right) & =\lim _{b \rightarrow \infty} M_{\mathrm{eq} 11}\left(b, R ; B_{z} / b, 0\right) \\
& =\left(\begin{array}{cccc}
1 & 0 & 0 & 0 \\
0 & 1 & B_{z} & 0 \\
0 & 0 & 1 & 0 \\
-B_{z} & 0 & 0 & 1
\end{array}\right) .
\end{aligned}
$$

From Eq. (25), one can see that for the delta fringe the transfer matrix is independent of the quadrupole gradient. The transfer matrix from $z$ to 0 can be obtained with that from 0 to $z$ according to the following:

$$
\begin{aligned}
M_{\mathrm{eq} 11}(b, R ; 0, z) & =K M_{\mathrm{eq} 11}^{-1}(-b,-R ; z, 0) K, \\
\text { where } K & =\left(\begin{array}{cccc}
1 & 0 & 0 & 0 \\
0 & -1 & 0 & 0 \\
0 & 0 & 1 & 0 \\
0 & 0 & 0 & -1
\end{array}\right) .
\end{aligned}
$$

Equation (26) can be explained as changing the direction of motion from $0 \rightarrow z$ to $z \rightarrow 0$ leading to changing the signs of $b, R, x^{\prime}$, and $y^{\prime}$.
From Eq. (26), one can get the delta fringe matrix $z \rightarrow 0$

$$
\begin{aligned}
M_{\delta}\left(0, B_{z}\right) & =\lim _{b \rightarrow \infty} M_{\mathrm{eq} 11}\left(b, R ; 0, \frac{B_{z}}{b}\right) \\
& =\left(\begin{array}{cccc}
1 & 0 & 0 & 0 \\
0 & 1 & -B_{z} & 0 \\
0 & 0 & 1 & 0 \\
B_{z} & 0 & 0 & 1
\end{array}\right) .
\end{aligned}
$$

Considering a triangle-type solenoid superimposed constant gradient quadrupole magnet, i.e., the solenoid field linearly increases from 0 to $B_{z}$ (with a slope of $b$ ) and then drops to 0 with a delta function, the transfer matrix being

$$
M=\left(\begin{array}{cccc}
1 & 0 & 0 & 0 \\
0 & 1 & -b z & 0 \\
0 & 0 & 1 & 0 \\
b z & 0 & 0 & 1
\end{array}\right) M_{\mathrm{eq} 11}(b, R ; z, 0)
$$

By doing some complicated calculations, one can prove the transfer matrix in Eq. (28) satisfies the symplectic condition, i.e., $M$ satisfies $M^{T} J M=J$, where

$$
J=\left(\begin{array}{cccc}
0 & 1 & 0 & 0 \\
-1 & 0 & 0 & 0 \\
0 & 0 & 0 & 1 \\
0 & 0 & -1 & 0
\end{array}\right)
$$

For any complex shape solenoid field, one can divide the solenoid into many pieces $(n)$. Assuming the solenoid field for the $n+1$ points are $b_{0}, b_{1}, \ldots, b_{n}$, the lengths of $n$ pieces are $L_{1}, L_{2}, \ldots, L_{n}$, and the quadrupole strengths in $n$ pieces are $R_{1}, R_{2}, \ldots, R_{n}$. All $b_{i}, L_{i}$, and $R_{i}$ can be different. The transfer matrix is

$$
M=M(n, n-1) \cdots M(2,1) M(1,0),
$$

where $M(i, i-1)$ is the transfer matrix of the $i$ th piece as follows:

$$
\begin{aligned}
M(i, i-1)= & M_{\mathrm{eq} 11}\left(\frac{b_{i}-b_{i-1}}{L_{i}}, R_{i}, \frac{b_{i} L_{i}}{b_{i}-b_{i-1}}, \frac{b_{i-1} L_{i}}{b_{i}-b_{i-1}}\right) \\
= & M_{\mathrm{eq} 11}\left(\frac{b_{i}-b_{i-1}}{L_{i}}, R_{i}, \frac{b_{i} L_{i}}{b_{i}-b_{i-1}}, 0\right) M_{\mathrm{eq} 11}^{-1}\left(\frac{b_{i}-b_{i-1}}{L_{i}}, R_{i}, \frac{b_{i-1} L_{i}}{b_{i}-b_{i-1}}, 0\right) \\
= & M_{\delta}\left(b_{i}, 0\right)\left[M_{\delta}\left(0, b_{i}\right) M_{\mathrm{eq} 11}\left(\frac{b_{i}-b_{i-1}}{L_{i}}, R_{i}, \frac{b_{i} L_{i}}{b_{i}-b_{i-1}}, 0\right)\right] \\
& \times\left[M_{\delta}\left(0, b_{i-1}\right) M_{\mathrm{eq} 11}\left(\frac{b_{i}-b_{i-1}}{L_{i}}, R_{i}, \frac{b_{i-1} L_{i}}{b_{i}-b_{i-1}}, 0\right)\right]^{-1} M_{\delta}\left(0, b_{i-1}\right) .
\end{aligned}
$$


Substituting Eq. (31) into Eq. (30), one can get

$$
M=M_{\delta}\left(b_{n}, 0\right) M_{1, n} M_{0, n}^{-1} \cdots M_{1,1} M_{0,1}^{-1} M_{\delta}\left(b_{0}, 0\right),
$$

where

$$
\begin{aligned}
& M_{1, i}=M_{\delta}\left(0, b_{i}\right) M_{\mathrm{eq} 11}\left(\frac{b_{i}-b_{i-1}}{L_{i}}, R_{i}, \frac{b_{i} L_{i}}{b_{i}-b_{i-1}}, 0\right) \\
& M_{0, i}=M_{\delta}\left(0, b_{i-1}\right) M_{\mathrm{eq} 11}\left(\frac{b_{i}-b_{i-1}}{L_{i}}, R_{i}, \frac{b_{i-1} L_{i}}{b_{i}-b_{i-1}}, 0\right)
\end{aligned}
$$

Since Eq. (28) satisfies the symplectic condition, all the terms $M_{1, i}, M_{0, i}$ satisfy the symplectic condition except the two terms $M_{\delta}\left(b_{n}, 0\right), M_{\delta}\left(b_{0}, 0\right)$. When $b_{n}$ and $b_{0}$ are equal to $0, M$ is symplectic. So for any shape of the solenoid the transfer matrix is symplectic as long as the solenoid field is from 0 to 0 .

\section{ACKNOWLEDGMENTS}

The author would like to thank Professor A. Chao (SLAC) for his encouragement and helpful discussions.

[1] D. Rubin, in Handbook of Accelerator Physics and Engineering, edited by A. Chao and M. Tigner (World Scientific, Singapore, 1999), p. 269.

[2] C. X. Wang and A. Chao, SLAC Report No. SLAC/ AP-106, 1996.

[3] B. Nash, SLAC Report No. SLAC/AP-136, 2000.

[4] A. Dragt, Nucl. Instrum. Methods Phys. Res., Sect. A 298, 441 (1990).

[5] V.V. Andreev and I. P. Yudin, in Proceedings of the XVth International Conference on High Energy Accelerators, HEACC'92, Hamburg, edited by J. Rossbach (World Scientific, Singapore, 1993), Vol. II, p. 1013.

[6] M.W. Garrett, J. Appl. Phys. 34, No. 9, 2567 (1963).

[7] A. Erdelyi, Higher Transcendental Functions (McGrawHill, New York, 1953), Vol. I, p. 184. 